\title{
Antioxidant Synergistic Effect and Formulation Optimization of Several Common Natural Pigments
}

\author{
Yi-Hua JIANG ${ }^{1, a, *}$, Xin-Long JIANG ${ }^{2, b}$, Hui-Qiang BAO ${ }^{3, c}$ \\ ${ }^{1,3}$ Zhejiang Shuren University, Hangzhou, China \\ ${ }^{2}$ Institute of Food and Fermentation Engineering, Zhejiang Shuren University, Hangzhou, China \\ ahzjyih828@163.com, bjxinl2003@163.com, ${ }^{c} 291565780 @ q q . c o m$
}

${ }^{*}$ Corresponding author

Keywords: Antioxidant, Synergistic Effects, Response Surface Method (RSM), Gardenia Yellow, Black Bean Red, Sorghum Red.

\begin{abstract}
Under DPPH system using the method of free radical scavenging, synergetic effect and optimal formulation of compound antioxidant among the solution of gardenia yellow, black bean red and sorghum red with the response surface method were determined. The results showed that, the component ratio of optimal compound antioxidant was gardenia yellow $20.00 \mu \mathrm{g} \cdot \mathrm{ml}^{-1}$, black bean red $100.00 \mu \mathrm{g} \cdot \mathrm{ml}^{-1}$, sorghum red $300.00 \mu \mathrm{g} \cdot \mathrm{ml}^{-1}$, the DPPH scavenging rate of the system was $67.79 \%$, compound antioxidant of gardenia yellow, black bean red and sorghum red had synergistic effect .Through the comparison of optimal mixed antioxidants and single antioxidant, the mixed antioxidants were better than single antioxidant slightly.
\end{abstract}

\section{Introduction}

Antioxidants, the free radical eliminators, are the important issue of research in the medical profession and antiaging field. If we absorb efficient antioxidants enough through food intake, it can help us remove the excess free radicals in our bodies, improve health, delay the disease and prolong life.

Gardenia yellow pigment is which is extracted from Gardenia jasminoides Ellis and widely used as a natural food colorant in Asian countries. Gardenia yellow are carotenoids of peculiar water-soluble behavior[1].Sorghum red pigment is from the sorghum shell and safety,not poisonous edible.Main components of red pigment were 5,4'-two hydroxyl isoflavones-7-O-galactoside and 5,4'-two hydroxyl-6,8-two methoxy isoflavone 7-O-galactoside[2].The black bean red belongs to the anthocyanins, which is the main component of delphinidin -3-glucoside and delphinidin and cyanidin -3- glucoside[5],Sorghum red pigment is isoflavones galactoside,a kind of natural pigment from the sorghum shell and safety,not poisonous edible. Main components of red pigment were 5,4'-two hydroxyl isoflavones-7-O-galactoside and 5,4'-two hydroxyl-6,8-two methoxy isoflavone 7-O-galactoside[3].The pigments natural pigments showed a higher scavenging activity against free radicals such as DPPH·[1-3].

DPPH method is used to evaluate a natural antioxidant activity with sensitive, simple, rapid and feasible [4-5]. The gardenia yellow, black bean red and sorghum red as typical representative of natural pigments, the DPPH. scavenging rate as evaluation index of antioxidant activity, the synergetic effect and optimal formulation were determined using the response surface optimization method under DPPH. scavenging system in this paper.

\section{Material and Methods}

\section{Materials, Reagents}

Black bean red pigment (content 27.29\%) ,gardenia yellow pigment (content 13.60\%) and sorghum red pigment (content 41.32\%) were purchased from Qianjiang green sen treasure bio-tech Co., ltd.DPPH. was purchased from Sigma company, other reagents were of analytic grade. Distilled 
water was used throughout the experiment.

\section{Antioxidant Test}

DPPH radical-scavenging activity was performed by the method described of Ying QL [5]. The stock solution of DPPH. was prepared by dissolving $25.63 \mathrm{mg}$ in $100 \mathrm{ml}$ absolute ethyl alcohol $\left(6.5 \times 10^{-4} \mathrm{~mol} / \mathrm{L}\right)$. The stock solution was diluted to a dilution to $6.5 \times 10^{-5} \mathrm{~mol} / \mathrm{L}$ with $60 \%(\mathrm{v} / \mathrm{v})$ ethanol for each determination. An aliquot of each dilution $(1.0 \mathrm{~mL})$ was mixed with solution of $\mathrm{DPPH} \cdot\left(5 \mathrm{~mL}, 6.5 \times 10^{-5} \mathrm{~mol} / \mathrm{L}\right)$. The mixtures were shaken vigorously and incubated at $37{ }^{\circ} \mathrm{C}$ in the dark for $30 \mathrm{~min}$. A control containing $60 \%$ (v/v) ethanol $(1.0 \mathrm{~mL})$ and solution of DPPH $(5 \mathrm{~mL}, 6.5 \times$ $\left.10^{-5} \mathrm{~mol} / \mathrm{L}\right)$ was run at the same time. The absorbance was measured at $517 \mathrm{~nm}$ against $60 \%(\mathrm{v} / \mathrm{v})$ ethanol $(5.0 \mathrm{~mL})$ mixed tested extract solution $(1.0 \mathrm{~mL})$ as a blank. The percentage of DPPH scavenging was calculated as follows: $\mathrm{P} \%=\left[1-(\mathrm{Ai}-\mathrm{Aj}) / \mathrm{A}_{0}\right] \times 100$, where $\mathrm{Ai}$ is absorption of tested extract solution, $\mathrm{Aj}$ is absorption of blank, $\mathrm{A}_{0}$ is absorption of control.

\section{Synergy Analysis Method}

The effect produced exceeds the sum of their respective alone when used in combination of several substances, the synergistic effect is produced. The synergy evaluation method of antioxidant in vitro have three methods commonly: the response surface method, the addition method and the direct comparison method.RSM can optimize process parameters by combination of statistics and mathematics method and fit the factors and response value by quadratic regression equations[6].Comparison of the response coefficients in the equation, can be quantified analysis factors influence on the composite antioxidant activity[7]. The subject chosen response surface methodology (RSM).

\section{Response Surface Test Method}

\section{The Single Factor Test}

The sample solution $(1 \mathrm{mg} / \mathrm{ml})$ was dissolved with water. The sample solution was mixed with DPPH.solution $\left(5 \mathrm{~mL}, 6.5 \times 10^{-5} \mathrm{~mol} / \mathrm{L}\right)$. To add a small amount the sample solution at first, increases gradually, and observe the fade of solution while still mixing. When the solution basic color faded, remembered the maximum concentration. Based on the maximum concentration, set up the appropriate gradient and make arithmetic sequence. According to the antioxidant test method of single factor tested.

\section{The Response Surface Experimental Design}

According to the single factor experiment results, response surface methodology experiments of Box-Behnken design were chosen for formulation optimization. The 3 factors and 3 levels were listed in Table 1.

Tab. 1 Levels of factors in response surface methodology

\begin{tabular}{|l|c|c|c|}
\hline \multirow{2}{*}{ factors } & \multicolumn{3}{|c|}{ levels } \\
\cline { 2 - 4 } & -1 & 0 & 1 \\
\hline A gardenia yellow $\left[\mu \mathrm{g} \cdot \mathrm{ml}^{-1}\right]$ & 20 & 30 & 40 \\
B Sorghum red $\left[\mu \mathrm{g} \cdot \mathrm{ml}^{-1}\right]$ & 100 & 200 & 300 \\
C Black bean red $\left[\mu \mathrm{g} \cdot \mathrm{ml}^{-1}\right]$ & 60 & 80 & 100 \\
\hline
\end{tabular}

\section{Result and Analysis}

\section{The Single Factor Test}

The DPPH- Scavenging Rate of the Gardenia Yellow. Figure 1 showed, the ability of scavenging DPPH increased with increasing concentration in the $70 \mu \mathrm{g} / \mathrm{ml}$ range. The change of the 
scavenging DPPH ability tended to be stable after $70 \mu \mathrm{g} / \mathrm{ml}$. Three levels were of $20,30,40 \mu \mathrm{g} / \mathrm{ml}$ were determined at gardenia yellow concentration.

The DPPH• Scavenging Rate of the Sorghum Red. Figure 1 showed, the ability of scavenging DPPH increased with increasing concentration and it had a high dose effect relationship in the range of $800 \mu \mathrm{g} / \mathrm{ml}$. Three levels were of $100,200,300 \mu \mathrm{g} / \mathrm{ml}$ were determined at sorghum red concentration.

The DPPH· Scavenging Rate of the Black Bean Red. Figure 1 showed, the ability of scavenging DPPH increased with increasing concentration in the range of $160 \mu \mathrm{g} / \mathrm{ml}$. The change of the scavenging DPPH ability tended to be stable after $160 \mu \mathrm{g} / \mathrm{ml}$. Three levels were of $60,800,100 \mu \mathrm{g} / \mathrm{ml}$ were determined at black bean red concentration.
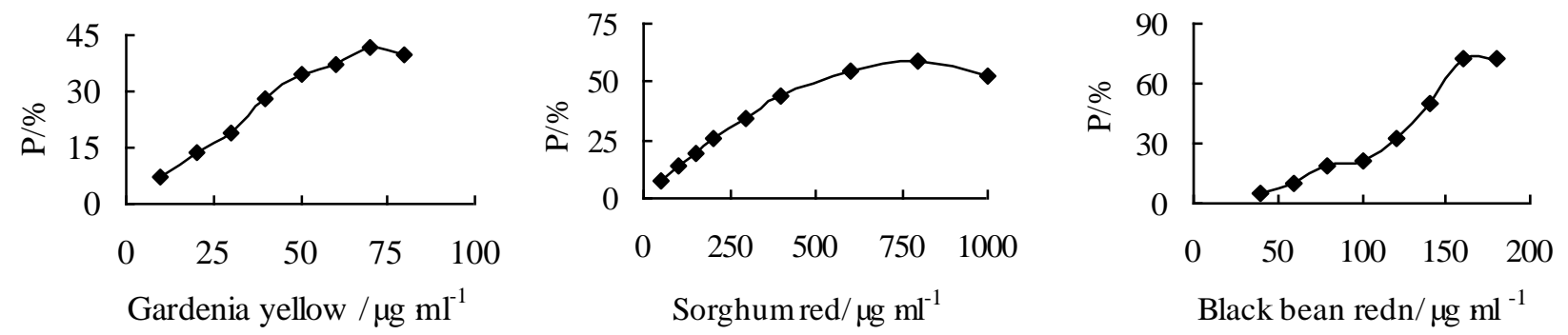

Fig.1 Effects of different factors on the DPPH· scavenging rate

\section{The Test Results of RSM}

Based on the results of single factors experiments, the formulation optimization of gardenia yellow, black bean red and sorghum red experiments were carried out by RSM design according to Table 2 .

Tab. 2 Data of RSM

\begin{tabular}{ccccc}
\hline No. & $\mathrm{A}$ & $\mathrm{B}$ & $\mathrm{C}$ & $\mathrm{P} / \%$ \\
\hline 1 & 0 & 1 & 1 & 59.86 \\
2 & -1 & 0 & -1 & 25.09 \\
3 & 0 & -1 & 1 & 51.18 \\
4 & -1 & 0 & 1 & 52.18 \\
5 & 1 & 0 & 1 & 52.36 \\
6 & 0 & 0 & 0 & 55.49 \\
7 & 0 & -1 & -1 & 17.99 \\
8 & 1 & 1 & 0 & 25.09 \\
9 & 0 & 0 & 0 & 50.91 \\
10 & 0 & 0 & 0 & 43.53 \\
11 & 0 & 0 & 0 & 57.88 \\
12 & 1 & 0 & -1 & 42.15 \\
13 & 0 & 1 & -1 & 24.45 \\
14 & 1 & -1 & 0 & 56.91 \\
15 & 0 & 0 & 0 & 53.3 \\
16 & -1 & 1 & 0 & 57.42 \\
17 & -1 & -1 & 0 & 34.98 \\
\hline
\end{tabular}

\section{Analysis of Variance}

Analysis of variance was carried out by design expert and the results showed that the $\mathrm{p}$ values of black bean red (C), the interaction among gardenia yellow(A) and sorghum red (B) were less than 0.01, which showed that the factors had most important influence.Analysis of variance showed that the factors and responses were not linear relationship. Above results showed that the specific factors and scavenging response did not have simple correlation relations. 
Tab. 3 Analyze of mean square

\begin{tabular}{cccccc}
\hline $\begin{array}{c}\text { Sourc of } \\
\text { Squares }\end{array}$ & $\begin{array}{c}\text { Sum of } \\
\text { Square }\end{array}$ & $\begin{array}{c}\text { Degree of } \\
\text { freedom }\end{array}$ & $\begin{array}{c}\text { Mean } \\
\text { Square }\end{array}$ & F Value & Prob > F \\
\hline Model & 2680.64 & 9 & 297.85 & 5.03 & 0.0223 \\
A & 5.85 & 1 & 5.85 & 0.099 & 0.7624 \\
B & 4.15 & 1 & 4.15 & 0.07 & 0.7988 \\
C & 1401.85 & 1 & 1401.85 & 23.69 & 0.0018 \\
AB & 736.04 & 1 & 736.04 & 12.44 & 0.0096 \\
AC & 71.23 & 1 & 71.23 & 1.2 & 0.3089 \\
BC & 1.23 & 1 & 1.23 & 0.021 & 0.8893 \\
A $^{2}$ & 17.24 & 1 & 17.24 & 0.29 & 0.6061 \\
B $^{2}$ & 183.33 & 1 & 183.33 & 3.1 & 0.1218 \\
C $^{2}$ & 221.53 & 1 & 221.53 & 3.74 & 0.0943 \\
Residual & 414.24 & 7 & 59.18 & & \\
Lack of Fit & 293.11 & 3 & 97.7 & 3.23 & 0.1437 \\
Pure Error & 121.13 & 4 & 30.28 & & \\
Cor Total & 3094.88 & 16 & & & \\
\hline
\end{tabular}

Fitting the Model to Establish and Antioxidant Synergy Analysis

To fit the response surface test data, to obtain the coding variable regression equation of two order polynomia.

$$
\mathrm{P}=+52.22+0.85 \mathrm{~A}+0.72 \mathrm{~B}+13.24 \mathrm{C}-13.57 \mathrm{AB}-4.22 \mathrm{AC}+0.56 \mathrm{BC}-2.02 \mathrm{~A}^{2}-6.60 \mathrm{~B}^{2}-7.25 \mathrm{C}^{2}
$$

The $p$ vale of the lack of fit value was of 0.1437 (not significant), the model was of 0.0223 (significant), which showed that the model fit the RSM data significantly, the equation was fit the synergetic antioxidant effect among the gardenia yellow, black bean red and sorghum red.

According to the regression equation, the first degree were always positive, indicating that gardenia yellow, black bean red and sorghum red were positive correlation with the scavenging rate of DPPH. The interaction coefficient of gardenia yellow (A) and sorghum red (B) was negative value, reflecting the synergistic effect between two factors was negative cooperativity. Negative cooperativity effect of gardenia yellow (A) and black bean red (C) was in the same way.The interaction coefficient of sorghum red (B) and black bean red (C) were positive value, reflecting the synergistic effect between two factors was positive cooperativity. Compound antioxidant of gardenia yellow, black bean red and sorghum red had synergistic effect by and large .

Using the antioxidants concentration of gardenia yellow, black bean red and sorghum red as $\mathrm{X}$ and $\mathrm{Y}$, the scavenging rate of $\mathrm{DPPH}$-as the $\mathrm{Z}$, make the corresponding 3D figure. The interaction between gardenia yellow(A) and sorghum red (B) had great influence on the response, which showed relatively steep curve in the figures and it was consistent with the regression analysis results.

\section{The Optimal Process Validation}

The maximum value of model was calculated by the software. The equivalent linear regression model was shown in Figure 2.The component ratio of optimal compound antioxidant was gardenia yellow $20.00 \mu \mathrm{g} \cdot \mathrm{ml}^{-1}$, black bean red $100.00 \mu \mathrm{g} \cdot \mathrm{ml}^{-1}$, sorghum red $300.00 \mu \mathrm{g} \cdot \mathrm{ml}^{-1}$, the $\mathrm{DPPH}$. scavenging rate was $67.79 \%$, the verified value could reach above $67.5 \%$, it was consistent with the predicted value and verified the validity of the model. The single component test with combination formulations of gardenia yellow $20.00 \mu \mathrm{g} \cdot \mathrm{ml}^{-1}$, black bean red $100.00 \mu \mathrm{g} \cdot \mathrm{ml}^{-1}$, sorghum red $300.00 \mu \mathrm{g} \cdot \mathrm{ml}^{-1}$, the actual production of DPPH. scavenging rate were: $13.62 \% \%, 20.83 \%$ and $32.33 \%$, respectively. The total scavenging rate was $66.78 \%$,it was less than the combined formula slightly.It showed that combined formula antioxidant activity was better than single antioxidant and consistent with the regression model analysis. 


\section{Conclusion}

In this study, in order to understand synergetic antioxidant effect of several common natural pigments among the gardenia yellow, black bean red and sorghum red, synergetic effect and optimal formulation of compound antioxidant were determined with the response surface method. The optimum formula of compound antioxidants were : gardenia yellow $20.00 \mathrm{~g} \cdot \mathrm{ml}^{-1}$,black bean red $100.00 \mu \mathrm{g} \cdot \mathrm{ml}^{-1}$, sorghum red $300.00 \mu \mathrm{g} \cdot \mathrm{ml}^{-1}$, the DPPH scavenging rate was $67.79 \%$ under the conditions. Comparison the antioxidant activity between single antioxidant and compound antioxidants, concluded that compound antioxidants slightly better than single antioxidant. In the concentration, there were synergistic effect of antioxidant.
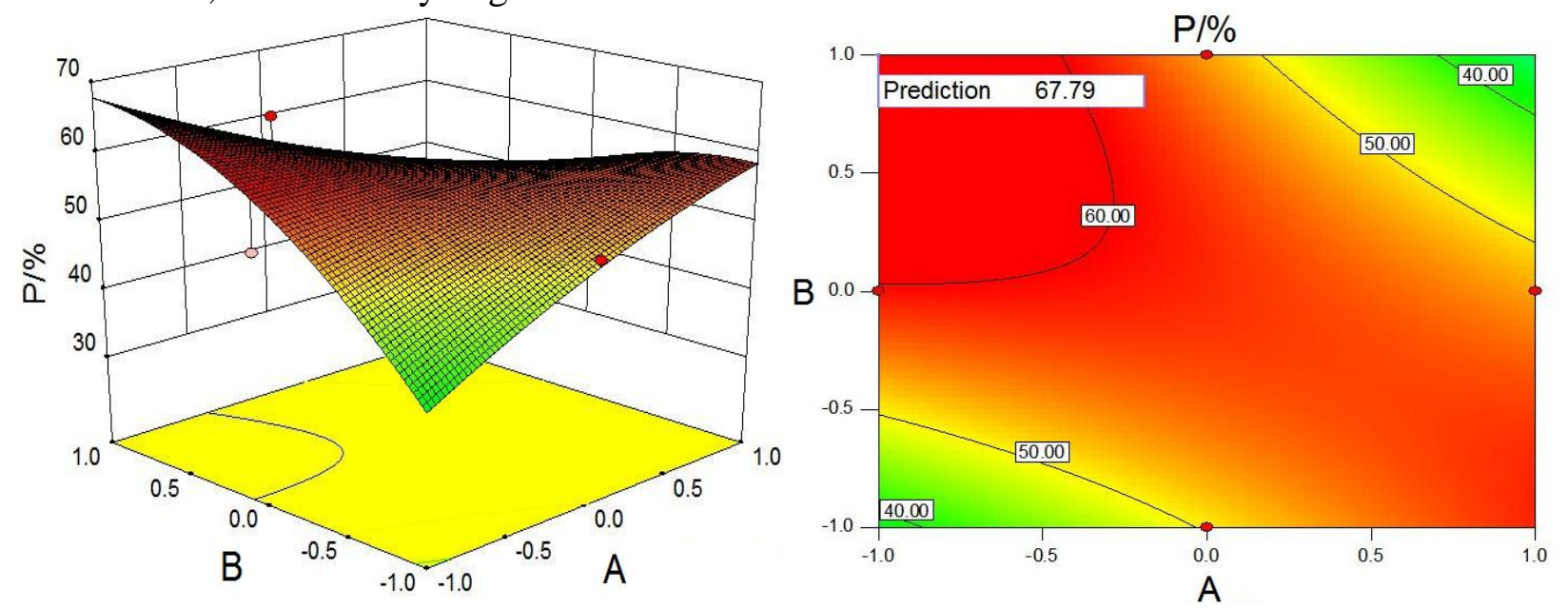

Fig.2 The equivalent linear regression model and $\mathrm{P}=\mathrm{f}(\mathrm{A}, \mathrm{B})$ response surface

\section{Acknowledgments}

This work was supported by the Program of Science and Technology of Zhejiang Province, China (No.2014C31138) .

\section{References}

[1] Li Xiong,Study on the Extraction and Antioxidant Activity of Gardenia Yellow Pigment[D].Changsha:Central South University of Forestry\&Technology.2012.

[2] Wang $\mathrm{J}$ T,Research advances on natural red pigment form sorghum bicolor[J]. Cereals \& Oils,2012(11):7-11.

[3] Li L R,Zhang M W,Liu L W,Comparing the stability of three kinds of black cereal and oil crops seed coat anthocyanins extract antioxidant capacity[J].Agricultural science of Chinese, 2007,40(9):2045-2052.

[4] Okawa M,Kinjo J,Nohara T,et a1.DPPH (1,1-dipheny-2-picrylhydrazy1)radical scavenging activity of flavonoids obtained from some medicinal plants[J].Biol Pharm Bull,2001,24(10):1202-1205.

[5] Ying Q L,Study on debinding and antioxidant technology eel[D].Hzngzhou:Zhejiang Gongshang University.2011.

[6] S.J. Kalil,F.Maugeri,M.I.Rodrigues. Response surface analysis and simulation as a tool for bioprocess design and optimization[J].Process biochemistry, 2000,35:539-550.

[7] Tan Z Q,Wu W L,Pan L Y,Research on Compound Antioxidants on low energy of cocoa butter equivalent antioxidant[J].Science and technology of food industry,2012,33(19):185-187,291. 